\title{
El servicio de abastecimientos en el Municipio español
}

Es indudable que este tema es de actualidad, en virtud del Decreto de 30 de agosto pasado, por el que se revierten a los Municipios ciertas facultades que competían a la Comisaría General de Abastecimientos y Transportes, facultades que, seguramente, habrá hecho experimentar a Corporaciones y Alcaldes la sensación de agobio propio del que siente sobre sí una nueva responsabilidad; debido a que las Corporaciones se habían acostumbrado, o por el contrario, no habían detentado facultades que, siempre, tradicionalmente, habían sido objeto de la competencia local; pero que fueron relevadas de las mismas parcialmente, por el organismo citado. El agobio de que hablamos no lo experimentarían si detenidamente consideraran las atribuciones que el Municipio español ha tenido a través de su glorioso pasado, tan lleno de múltiples facetas, que bien pueden servir de guia, aún en los tiempos modernos, ya que el cometido y problemas que el Municipio hoy tiene, no son más que repetición de aquellos problemas que en otros tiempos preocuparon a las Corporaciones y que están dentro de su competencia; el problema que nos ocupa no ha dejado de ser uno de los principales, pues como dice Desdevises du Dezert, "La cuestión del aprovisionamiento de las ciudades ha sido en todos los tiempos el principal asunto de los Municipios" (1). Por tanto, creemos oportuno hacer un leve bosquejo de las atribucio-

(1) Desdevises du Dezert, L'Espagne de l'Ancien Regime. 
nes y servicios que en materia de abastos han tenido las Corporaciones locales a través de su fecunda historia, alejándonos del detalle prolijo, aunque sin dejar de hacer un estudio sistemático, ya que no podemos contar con espacio para una detenida exposición.

Durante la Reconquista.-Epoca foral.

En la ordenación general del Municipio de esta época, de la que tenemos múltiples testimonios escritos e incluso recopilaciones y textos originales de Fueros, encontramos entre ellos numerosas disposiciones relativas a atribuciones $\mathrm{y}$ fines sobre lo que es objeto de nuestra atención: Abastecimiento público (2).

Cualquiera que sea el Fuero que nos detengamos a examinar, en él encontramos este servicio ocupando lugar preferente. ¿Es que en aquella época existía ya el problema? Indudablemente; jamás fué ni viejo ni nuevo, ha sido y es ell problema que constituye una preocupación constante para las autoridades, principalmente las locales, por ser éstas las que más intimamente se encuentran afectadas por sus consecuencias, dada su proximidad con el mismo. La autoridad local, debido a su contacto directo con el pueblo, aprecia en toda su magnitud los problemas que le afectan y cuáles de ellos son de más necesidad atender preferentemente; pues bien, este problema del abasto público lo vemos reflejado en todos los Fueros del medievo, y aunque no hay una absoluta paridad en ellos respecto a las diversas facetas del servicio, sí hay unanimidad en darle idéntica importancia y en muchas disposiciones coinciden.

Considerando como primer jalón de este modesto estudio el

(2) S. Mrngurjón, Historia del Derecko Español, cuaderno III, 3." edición, página 108.

E. DE Hinojosa, Organización del Munnicipio Español en la Edad Media (León y Castilla), 1903, pág. 20. 
Fuero de León (1920), en él vemos ya delimitado el servicio de abastecimientos en su cometido de inspección, fiscalización y señalamiento de tasas, función encomendada al Concejo y por delegación del mismo, a los Jurados, como podemos ver en el Capítulo 29 del Fuero, que dice que reunido el Concejo en $\mathrm{Ca}$ pitulo "et constituat mensuras panis et vini et carnis et pretium laboratium qualite omnis civitas teneat instition in ello anno" (3).

Más tarde, en 1266, un documento testifica la vigencia de este mismo Capítulo del Fuero: "Que fuero y era de León et costumbre guardada de cincuenta años acá, et del tiempo que ome non se podía acordar que el Cabildo de la Iglesia de León et el Concejo de ese mismo lugar se ayuntaban cada año el primer: Viernes de Quaresme en la calostra de Santa María de Regla, et facían sus posturas en qual manera debiese avenir todo el año, en razón de las medidas del pan, et del vino, et del precio de las carnes, et de los pescados, et el jornal de los obreros, et de la guarda de las viñas, et ponían comunalmente Jurados porque estas posturas fuesen mantenidas et guardadas" (4). Asimismo en el Fuero de Nájera (1001-1035) vemos preceptos sobre abastecimientos, confiriendo al Concejo atribuciones sobre los mismos y regulando pesas, medidas, precios y estableciendo medidas de vigilancia (5). En el de Oviedo, el Concejo también entendía en la policía de abastos y mercados, según las Ordenanzas de 1245-1274 (6).

Son múltiples las facetas que ofrecen los Fueros dignas de tenerse en cuenta, mas la índole de este trabajo no nos permite más que enumerar algunas de ellas para poder dar una idea de hasta qué punto intervinieron los Concejos en los abastecicimientos; así vemos como en el Fuero de León, se confía al Concejo la facultad de dar licencia para la venta de carne y penar las infracciones que se cometieren en el abasto público,

(3) Muñoz, Colección de Fueros, pág. 68.

E. DE Hinojosa, obra citada, pág. 20.

(4) P. Flores, España Sagrada, tomo XXXV, pág. 436.

E. Hinojosa, obra citada, pág. 20.

(5) MuÑoz, obra citada, pág. 290.

(6) Vigil, Colección histórico-diplomática, págs. 40 y 64-69. 
por falta de peso, calidad o precio de los artículos; la carne, antes de ponerla a la venta, se había de dar a prueba al Concejo. El Fuero de Cuenca confía la inspección de pesas y medidas al Almotacén.

Muestran especial predilección los Fueros en dar instrucciones sobre la elaboración, peso, clase y cochura del pan; de la carne y del vino. El de Cuenca dice: "El Almotacén debe ser sobre todas las medidas de pan, e de vino, e de olio, e de sal et sobre los pesos y libras de los carniceros et sobre los panaderos...", siendo larga la enumeración que continúa haciendo, mayor que la del Fuero de León, pues cdesde el Fuero de León (1020) hasta el de Cuenca (Alfonso VIII, sin fecha) se nota un progreso extraordinario" (7), y por tanto, los preceptos en él contenidos tenían que ser más minuciosos y amplios.

Pero no sólo a las Corporaciones les estaban conferidas facultades sobre los abastos, sino también, como dejamos dicho; a los Jurados o Fieles (8), por delegación y con jurisdicción directa, "Correspondía a los Alcaldes la policía rústica y urbana, fijar la cabida o mano legal de las pesas y medidas, tasar cada año los jornales y los precios de varios artículos de consumo" (9).

Más tarde, en el reinado de Alfonso XI, en Sevilla aparecen más recargadas las disposiciones por las que se ha de regir la ciudad, y por tanto, en materia de abastos, no deja de suceder otro tanto. Por eso tienen los Almotacenes más facultades: "que pesen el pan en los fornos, et fuera de los fornos do quier que fallaren a vender; et el pan que fallaren menguado, o mal cocho, o olivado, aquí en la Ciudat, que sea para los presos de la cárcel, et en los lugares de los términos, que sea para los Almotacenes" (10).

Asimismo se determinan las condiciones que habían de re-

(7) D. Romero, conferencia sobre aNormas de policía urbana en los fueros municipales», 1930

(8) Hinojosa, obra citada, pág. 118.

(9) A. SACRIStán, Municipalidades de Castilla y León, págs. 262-264.

(10) Guichot y Parody, Historia del Ayuntamiento de Sevilla, tomo I, página 250 (Ordenanzas del Concejo). 
unir los ciudadanos nombrados para los cargos, y entre ellos, por ser propios de la materia que nos ocupa, los Almotacenes y Alamines: "... que los Alcaldes Mayores pongan dos omes buenos quantiosos et de buena forma, por Alamines, porque guarden bien et verdaderamente la fialdat con que son puestos; et si non lo ficieren, que sean echados por malos de los oficios, et donde adelante que nunca ayan oficion (11); para la designación de Ailmotacenes señala idénticas condiciones. Las facultades de estos funcionarios se encuentran detenidamente reguladas; respecto al pan, dice el mismo ordenamiento que, los Alamines den cada lunes y jueves a los Almotacenes "las pesas del pan derechas, según que fuere el precio de la farina", la que también se interviene en su cernido, en evitación de que se adultere su calidad; "los Alcaldes Mayores que den padrones ciertos a los atofaneros, porque ciernan la farina segunt se debe cernern.

No sólo la cantidad y calidad era motivo de vigilancia, sino que el precio de los productos era intervenido y tasado por el Concejo, como vemos en las Ordenanzas de Sevilla, mandadas recopilar por los Reyes Católicos (12), lo que nos demuestra que el intervencionismo, por parte de la Autoridad, en materia de abastos, no es una novedad, ya que tanto estas disposiciones como otras anteriormente citadas, más antiguas, así lo manifiestan, haciéndonos pensar que ello sería debido a la escasez de ciertos productos; y en evitación de especulaciones, adoptaban tales medidas, o por el contrario, aun habiendo abundancia, el sistema de tasas se aplicaba a los alimentos de primera necesidad, de uso común entre la clase pobre u obrera en evitación de su encarecimiento por parte de desaprensivos.

\section{Durante la Edad Moderna.-Epoca centralizadora.}

Ya en los últimos tiempos de la época anterior, se iba experimentando una evolución del Municipio, al que se van restando facultades por el Poder central con detrimento notable

(11) Ordenamiento de Alfonso $X I$, de 1337, para los Alamines y Almotacenes.

(12) Gurchot y PARodi, obra citada, tomo I, pág. 316 (Ordenanza XLI). 
para la Institución; estos síntomas centralizadores se fueron haciendo más intensos en los reinados siguientes.

En esta época en que la centralización es la característica administrativa más acusada, no podía quedar al margen el problema de abastecimientos, de esa política general.

Así en esta fase del Municipio español y, concretamente en el servicio de abastos, vemos que el Corregidor, funcionario de designación real, tiene una posición preponderante, como podemos apreciar en la Pragmática de 1500 (Cap. 17, 19 y 32), que señala su competencia en materia de abastos y policía urbana; vigilancia del cumplimiento de las Ordenanzas municipales y formación de nuevas (13); disposición que más tarde fué reflejada en la Instrucción de 1713, para el ejercicio del cargo de Corregidor de Aragón y Valencia, en la que se encomienda a los Regidores la fijación de los precios de artículos, y a los Corregidores, la vigilancia y sanción de las contravenciones.

Dice Albi (14) que "la materia de abastos siguió en importancia a la administración de Propios, constituyendo con ella las dos funciones básicas del Municipio den la Monarquía absolutan; esta paridad en importancia de tales cometidos municipales tiene una justificación bastante elocuente, ya que el fomento y buena administración de los Propios hacía incrementar la riqueza agrícola y ganadera, repercutiendo en la mejora de los abastecimientos.

La libertad en el abasto público había sufrido un colapso y todo se encontraba intervenido, diciendo Albi que era cuna economía intervenida de tipo rudimentarion y los Regimientos actúan en la regulación de los artículos destinados al consumo público con plenitud de atribuciones (15); igualmente encontra-

(13) F. ALBI, El Corregidor en el Municipio español bajo la Monarquía ábsoluta, pág. 59.

(14) F. AlBr, obra citada, pág. 164.

(15) F. AlBI, obra citada, pág. 165. 
mos en Santallana (16) y Ortiz de Zúñiga (17), y debido a este intervencionismo las Ordenanzas municipales regulaban prolijamente la materia de abasto, pero no sólo los artículos de primera necesidad, sino también otros, como veremos más adelante en alguna Ordenanza que citaremos como ejemplo. Pero lo fundamental de la intervención consistía en la tasa de los artículos de primera necesidad; subastas para conceder exclusivas al abasto de la población, a los precios que resultaren en el remate y municipalización de otros; ya en esta época también se sintió. le necesidad, además de tasar los productos, de regular su mercado directamente por la Autoridad.

Se dispuso por Cédula de 16 de junio de 1767 la libertad en el comercio de las subsistencias, siendo muy corta la vigencia de tal disposición, pues se volvió al sistema en 1768 (18), parcialmente, y en su totalidad en 1772 (19). En este intervencionismo tienen acción directa las Corporaciones juntamente con el Corregidor, cuya autoridad predomina; esta cooperación se efectúa a través de una comisión integrada por el Corregidor y dos o cuatro Diputados nombrados por el Concejo (20) y los que, dada la preponderancia del Corregidor, quedan relegados a meros consejeros o asesores, hecho que nos manifiesta, una vez más, cómo el Poder central iba absorbiendo lenta, pero continuamente, la autonomía de los Municipios.

No queremos pasar más adelante en nuestra exposición sin citar antes como ejemplo de la épocá que acabamos de consisiderar, unas Ordenanzas bastante curiosas que nos dicen de una manera concreta hasta qué punto llegó el intervencionismo: las "Ordenanzas de Burgos" (21).

(16) Santallana, Gobierno político de los pueblos de España y el Corregidor: Alcalde y Juez de ellos, págs. 142-143.

(17) Ortíz de Zúñiga Y Herrera, Deberes y atribuciones de los Corregido res, Justicias y Ayuntamientos de España, pág. 164.

(18) aProvisión del Concejon, de 2 de noviembre de 1768.

(19) Provisión del Concejo,, de 11 de mayo de 1772.

(20) F. Albi, obra citada, pág. 167.

(21) Ordenanzas de Burgos», de 3 de febrero de 1747. Madrid, imprenta Ma. nuel Fernández. 
En estas Ordenanzas vemos con cuánto detenimiento se regulaba el comercio, no sólo de los productos de primera necesidad, sino de otros muchos, dedicando a cada uno varias disposiciones y quedando por tanto reglamentado hasta en el detalle más pequeño. El abasto de vino (22) estaba sometido a diversas condiciones para ser otorgada la concesión, respecto a la forma, calidad, vigilancia, etc. El pescado (23) había de ser vendido por aquel que obtuviera la concesión por un año, la cual se otorgaría: cal que hiciere postura más favorable a la utilidad del pueblon; el pescado de río no podía ser comprado en la ciudad para ser revendido, norma esta, al parecer, que iba contra la especulación.

Respecto al pan (24), indica los lugares donde este artículo se había de vender por los que de otros pueblos surtían a la ciudad, señalando el peso que cada pieza había de tener, condiciones en que lo habían de llevar, etc., "vendiendo cada cosa por lo que sea; esto es, pan de trigo, por pan de trigo; centeno, por centeno; y asi de las demás semillas". En la Ordenanza 164 señala la forma y peso de las piezas para "el abasto de gente pobre, y trabajadora", impidiendo se hicieran "hogazas de ochenta y cinco onzas... ya para evitar el daño que comúnmente sed padecía de no cocer bien, siendo de tan crecido tamaño, ya porque más fácilmete pueda surtirse el pobre».

El mercado de fruas (25) lo regula detenidamente, desde la introducción en 'ell mercado-no permitiendo los intermediarios-, hasta las condiciones de los frutos, así como el destino que se había de dar al sobrante una vez dadas las doce del día, que podía ser vendido a las "regatonas"; idénticas prescripciones señala para las hortalizas, las que ninguna persona que se dedique a la venta de ellas puede comprarlas en las huertas del término de la ciudad, ni los hortelanos venderlas a estas personas antes de las doce del día, y los que las

(22) Ordenanzas de Burgos», ord. 124 a 137.

(23) Ordenanzas de Burgos», ord. 138 a 142.

(24) Ordenanzas de Burgosø, ord. 160 a 186

(25) Ordenanzas de Burgos», ord. 188 a 194. 
vendieran en las huertas había de ser "a la postura que en la Plaza se hicieren.

También se encuentra regulada la misión de los funcionarios (26) encargados de la fiscalización y vigilancia en materia de abastos; los Jueces de Fiscales han "de entender, y entiendan, según que así se ha practicado en todo lo concerniente a los abastos públicos de la ciudad y mantenimientos, que vinieren a venderse a ella, moderando los agravios y excesos que hubiere y se hallaren", regulando seguidamente la misión de los funcionarios de una manera ordenada y minuciosa, Estos funcionarios estaban sujetos a la inspección de los Procuradores Mayores que debían "asistir a todos los abastos, Plaza y comercio, de cualquier género que sea porque en ellos se observe y guarde la conmutativa justicia... siendo celosos igualmente sobre que los Fieles cumplan con su ministerio y obligación». A los Fieles se les confiaba la vigilancia de los pesos y medidas, para que fueran exactos, así como el de hacer posturas de todos aquellos artículos que diariamente entraren en el mercado.

Determinaban también las Ordenanzas la forma de designación de los funcionarios y autoridades que habían de entender en los abastos: los Jueces de Fieles eran designados por turno entre los "Caballeros Regidores", de dos en dos meses; los Procuradores Mayores se nombraban por los Procuradores de Colación, en número de dos, debiéndoles dar posesión en el primer Ayuntamiento del año; su ejercicio era anual, pero podían ser reelegidos. $\mathrm{Y}$ los Fieles eran nombrados por el Concejo cada año, en número de cuatro, entre los vecinos. de la ciudad. Todos estos funcionarios tenían facultades delegadas del Concejo al que tenían que dar cuenta de la gestión.

\section{Siglo XIX.-Epoca Constitucional.}

Hemos visto cómo el servicio de abastos se encuentra íntimamente unido al Municipio, a través de las épocas estudiadas, pero ahora entramos a considerar un período de nuestra

(26) Ordenanzas de Burgos», ord. 33 a 87. 
Historia bastante movido, dado su carácter de transición de la Monarquía absoluta al sistema constitucional, aunque en ella predomina reiteradamente la idea y tendencias constitucionalistas.

Las Cortes de Cádiz señalan un jalón, en la vida política española, que hace alterar todo el sistema anterior, introduciendo los sistemas democráticos de elección de los hombres que habían de regir los destinos de la nación y de los pueblos por el cuerpo electoral; desapareciendo en absoluto los cargos políticos y representantivos por designación del 'Poder central, aunque con anterioridad, reinando Carlos III, "reinado muy favorable al bien de los pueblos-como dice el Conde de Toreno-, dispúsose en $\mathbf{1 7 7 6}$ que éstos nombrasen Diputados y Síndicos, con objeto, en particular, de evitar la mala administración de los abastos, teniendo voto, entrada y asiento en los Ayuntamientos, y dándole en años posteriores mayor extensión de facultades", pero "no habiendo arrancado la raíz del mal, trató la Constitución de descuajarla» (27), tendiendo por ello a una descentralización, debiendo atender tan sólo el Gobierno las cuestiones y problemas de carácter nacional, o que por su magnitud requerían la acción centralizada, así como los que sólo podían ser de su exclusiva competencia. En virtud de tales medidas, el servicio de abastos pasa en toda su extensión a depender de los Municipios, que por disposiciones y ordenanzas de los mismos se regulan debidamente, de conformidad con la Instrucción de 1813, que ordena: "Para procurar la comodidad del pueblo, cuidará el Ayuntamiento, por medio de providencias económicas, conformes con las leyes de franquicia y libertad, de que esté surtido abundantemente de comestibles de buena calidad" (28).

La restauración con Fernando VII hace retroceder nuevamente al sistema absolutista, pero el alzamiento de Cabezas de San Juan abre de nuevo el período constitucional, se cele-

(2i) Conde de Toreno, obra citada, tomo III, pág. 506.

(28) Instrucción para el gobierno económico-político de las provincias» de 18 de junio de 1813 . Cap. 1.॰, art. 5.. 
bran las Cortes de 1821 al 1823 y, concretándonos a la materia que nos ocupa, en este período se promulga la "Ley para el Gobierno económico-politico de las provincias" de 1823, la que en su artículo 90, respecto a los Alcaldes, dice: "Cuidarán por sí y por medio de los Corregidores, y Alcaldes y Ayudantes de barrio de que no haya fraude en el buen precio y medida de los géneros que se venden y señaladamente de las especies de comestibles y consumo que los tienen conocidos". Esta reforma, introducida con la anterior Ley, en la Administración local, no llegó a tener larga vigencia, pues como dice Posada: "La reforma del régimen local, elaborada en 1823, no tuvo el mejor éxito inmediatamente; corrió análoga suerte a la de 1812 y 1813. Nueva reacción violenta, con intervención armada de Francia, dió al traste con la Constitución restaurada de 1812 y con toda la labor de las Cortes de 1812 a 1823") (29), siendo por un Real Decreto de $1 .^{\circ}$ de octubre de este mismo año anulados todos los actos del Gobierno constitucional.

"La reorganización de los Ayuntamientos constituídos sobre base popular se produce en el reinado de Isabel II, mediante el Real Decreto de 23 de junio de 1835, sobre arreglo provisional de los Ayuntamientos, dictado en virtud de autorización de los Estamentos; tiene interés este Real Decreto, que es una verdadera ley orgánica de Ayuntamientos, en razón a ciertos principios que formula, algunos de los cuales son de los que se han incorporado a nuestro Derecho municipal (30). En este Real Decreto se concede especial atención a los abastecimientos señalando como facultades y obligaciones de los Alcaldes en el artículo 36 , número $4 .^{\circ}$, "Inspeccionar los pesos y medidas, y el estado de la salubridad de los comestibles y bebidas". Y entre las atribuciones de los Ayuntamientos, artículo 48, número 17 : "Formar las Ordenanzas municipales", atribución ésta amplísima y por la que se les confiere una facultad con la que cada Ayuntamiento regulaba en sus Ordenanzas aquellos extremos que por su peculiaridad merecían la aten-

(29) A. Posada, Evolución Legislativạ del Régimen local en España, pág. 107.

(30) A. Posada, obra citada, pág. 116. 
ción y ordenación por la Autoridad, ya que no todas las Corporaciones tendrían los mismos problemas de abastecimientos, dadas las particularidades propias de cada región.

En 1840 se promulga la Ley de 14 de julio: "De gobierno y atribuciones de los Ayuntamientos"; suspendida su ejecución por Decreto de la Regencia en $1 .^{\circ}$ de octubre del mismo año, fué puesto en vigor por Real Decreto el 30 de diciembre de 1843, en la que se conferían facultades a los Ayuntamientos y Alcaldes en la materia de abastos.

Igualmente en la Ley de 8 de enero de 1845 de "Organización y atribuciones de los Ayuntamientos", en el artículo 73, número $5^{\circ}$, señala las atribuciones de los Alcaldes: "Cuidar de todo lo relativo a la policía urbana y rural, conforme a las leyes, reglamentos y disposiciones de la Autoridad superior y Ordenanzas municipales"; y a los Ayuntamientos, en el artículo 81 , número $1 .^{\circ}$, le atribuye "la formación de las Ordenanzas municipales y reglamentos de policía urbana y ruraln. Podemos observar en estos artículos que las facultades que se confieren a los Ayuntamientos y Alcaldes son bastante amplias, ya que ellas quedan dentro de un marco tan impreciso y elástico como indican los conceptos de: policía urbana y rural, ordenanzas y reglamentos municipales; lo que nos sugiere la idea de que en estas facultades no estarían ausentes las relacionadas con los abastecimientos, como nos lo atestiguan todas las Ordenanzas municipales de aquella época.

En las Leyes Municipales de 20 de agosto de 1870 y 2 de octubre de 1877 vemos idénticas disposiciones, aunque en ellas no hay paridad en la numeración de los artículos, pero a pesar de esto sus contenidos no difieren esencialmente; y ya en esta Ley encontramos más amplia la enumeración de las atribuciones de Alcaldes y Corporaciones; en la de 1877, artículo 72, número $1 .^{\circ}$, apartado $5 .^{\circ}$, señala dentro de las atribuciones de los Ayuntamientos, "casas de mercados y mataderos"; en el apartado 6..$^{\circ}$, "Ferias y mercados". En el número 2. , "Policía urbana y rural, o sea, cuanto tenga relación con el buen orden y vigilancia de los servicios municipales establecidos...n. En el 
artículo 74, número $1 .^{\circ}$, "Formación de Ordenanzas municipales de policía urbana y rural".

No sólo las leyes de ordeanción general de los Municipios son las que contienen preceptos referentes a abastos, sino que también se dieron disposiciones concretamente encaminadas a la mejor regulación de este servicio, de las que citaremos algunas a modo de ejemplo, pues una detenida enumeración sería demasiado larga: Real Decreto "Instrucción de 16 de abril de 1816", dando normas sobre las subastas de abastos. Real Decreto de 18 de julio de 1819, "Prevenciones para evitar abusos en los puestos públicos». Siendo Ministro de Fomento don Javier de Burgos se dió el Decreto de 20 de enero de 1834, que establece la libertad en el tráfico, comercio y venta de los objetos de comer, beber y arder; aboliendo las tasas y posturas : Policía de mercados, mataderos, pero esta libertad sin coartar las atribuciones de la autoridad municipal en la vigilancia de pesas, medidas y salubridad, y deja sin el beneficio de la libertad de tasa el pan. $Y$ por último, la Real Orden de 4 de enero de 1887. dando orden a los Gobernadores y Alcaldes sobre el cuidado debido en la vigilancia para evitar adulteraciones en las harinas, vinos, etc.

\section{De. 1900 a 1936.}

Podemos considerar los primeros años del presente siglo, en lo que a la Administración Local respecta, como una prolongación de la época anterior, pues la Ley Municipal de 1877 rige hasta 1924; por tanto, creemos innecesario hacer exposición ni comentario de la misma, ya que quedan hechos anteriormente, pero no obstante, conviene considerar la importancia que durante la vigencia de tal Ley adquirieron las Ordenanzas municipales, estando dedicadas partes de ellas a la regulación del abasteçimiento, en el que hay entera libertad y la Autoridad se limita a su inspección velando por la normalidad, pero sin intervenir directamente en el comercio ni en 
los precios; sólo el pan es el que se encuentra tasado y regulado su comercio.

Claro que en todo el período que nos ocupa, es lógico que hubiera tales libertades en los abastecimientos, porque también había posibilidades para ello sin el peligro de acaparamiento y especulación desmesurada, debido a la absoluta normalidad que se disfrutaba en la producción y consumo, asi como en el comercio internacional, siendo por tanto innecesaria la regulación por las Autoridades del comercio de los productos de primera necesidad: Pero no porque hubiera normalidad y libertad en el comercio la autoridad estaba ajena al servicio, sino que las Corporaciones seguían regulando, vigilando e inspeccionando los abastecimientos de conformidad con las atribuciones que la Ley Municipal les confería, y lo que constituia la garantía del ciudadano ante la posible transgresión o abuso por parte del comerciante o abastecedores.

La vigencia de la Ley Municipal de 1877 cesa al promulgarse la nueva de 1924, Ley de incalculable valor jurídico, elaborada concienzudamente bajo la dirección del gran político y hombre de Estado, Calvo Sotelo, que nos legó en tal Ley las líneas inmutables por las que se han de regir las Corporaciones, habiendo conseguido con ella devolver al Municipio gran parte de su antiguo y glorioso esplendor. Esta Ley se vió rectificada, en parte, por la República, pero no pudo serla en su totalidad porque en ella se habían plasmado preceptos que no podían ser alterados sin atacar los principios básicos del Municipio. Tanto en la Ley de Calvo Sotelo, como en la de la República, se señalan atribuciones de Ayuntamientos y Alcaldes respecto a abastos, sin que entre ambas se encuentren diferencias en tal respecto; así el texto de la de 1935, atribuye a los Alcaldes en su artículo 83, número 10, "Dirigir la policía de subsistencias" y a los Ayuntamientos les señala de su competencia, en el artículo 102, apartado B); la "potestad de Ordenanzas: aprobación y aplicación de las Ordenanzas, reglamentos e instrucciones y bandos municipales". El apartado F), letra c), "abastos, mataderos, mercados, hornos, tablas y panaderías". En el artículo 144 vuelve a hacer referencia 
a las Ordenanzas municipales, diciendo: "Los Ayuntamientos, dentro del ámbito de su competencia, regularán, mediante Ordenanzas, todas aquellas materias respecto de las cuales las leyes no contengan preceptos ordenadores concretos, siempre que en aquéllos no se infrinjan o contradigan preceptos legislativos o reglamentarios.

En esta Ley se le conceden, más expresamente, atribuciones a los Ayuntamientos respecto a abastos, como podemos apreciar en los anteriores artículos, por lo que la autoridad local queda revestida de esas facultades que se le confieren, además de las que se le otorgan con la potestad de ordenanzas, quedando una vez más en virtud de preceptos legales, vinculado el servicio de abastos, en gran parte, a las Corporaciones para que velen por su normalidad y eficiencia.

La Ley, en el artículo 132, confiere a los Ayuntamientos la facultad de munipalizar servicios, "según los casos, con carácter de monopolio, libremente, o tan sóló con el de regulación, los servicios... de mataderos, mercados..." $Y$ en el artículo 133, "también podrán los Municipios explotar, pero sin carácter de monopolio, establecimientos de suministro de artículos alimenticios y de primera necesidad, como hornos, tablas, panaderías y otros similares..."

Ya vemos que no sólo limita la Ley la acción municipal, respecto a abastos, a la inspección y vigilancia, sino que tambén concede la facultad de municipalizar determinados aspectos del servicio, facultad conferida, seguramente, en prevención de anormalidades que puedan alterar el abasto público, en cuyo caso los Ayuntamientos se encuentran facultados para asumir directamente la realización del servicio.

También el Estatuto Municipal de 8 de marzo de 1924 señala facultades y atribuciones a los Ayuntamientos y Alcaldes, más extensas que las de la Ley (en los artículos 150 , número $3 .^{\circ} \mathrm{y}$ 12 ; 153 , número $7 .^{\circ}$, y 192 , número 16 ), que son complementarios de aquéllas. $\mathrm{Y}$ el "Reglamento sobre obras, servicios y bienes municipales" de 1924, en los artículos. 86 al 92, confiere a los Ayuntamientos numerosas atribuciones relacionadas con los abastos, como "policía de subsistencias, inspección y examen 
de los alimentos y la acción y vigilancia en los mataderos, mercados y establecimientos donde se expendan sustancias alimenticias", y el debido control sobre las existencias de productos alimeticios en el término municipal, locales donde se efectúan las transacciones, así como la instalación de básculas para el peso del ganado en vivo y sobre los mataderos municipales.

Las Ordenanzas municipales que fueron aprobadas por los Ayuntamientos de conformidad con la Ley de 1924, nos presentan una profusión de normas, mayor que en las Ordenanzas anteriores; algunas de estas Ordenanzas regulan prolijamente el comercio de numerosos productos, claro que sin señalar tasa para ellos, ya que existía la libre contratación, pero, no obstante, hemos de hacer notar la acción tan directa que los Ayuntamientos y Alcaldes tenían en el servicio, osiendo designado corrientemente un Concejal para que atendiera- el mismo, con facultad delegada del Alcalde, con el fin de hacer más eficiente su control.

De 1936 a 1946.

Esquilmadas las reservas por nuestra guerra, así como la deficietaria producción en varios años por malas cosechas y la falta de un normal comercio exterior, hicieron adoptar por el Gobierno medidas encaminadas a suplir las deficiencias de la producción, con preceptos reguladores del comercio de aquellos artículos de primera necesidad que escaseaban y no llegaran a faltar en absoluto; de aquí que el Gobierno sintiera la necesidad de crear un organismo que centralizara los servicios de abastecimientos, siéndolo por Ley de 30 de enero de 1938 y organizado por Decreto de $\mathbf{1 6}$ de febrero del mismo año, con el nombre de "Servicio Nacional de Abastecimientos y Transportes", pero tanto su organización como funciones, no respondían a las necesidades que se hicieron sentir con posterioridad; siendo suspendida la actuación de tal organismo por Ley de 10 de marzo de 1939, que crea la "Comisaría General de Abastecimientos y Transportes", dependiente del Ministerio de Industria y Comercio, y organizada en virtud de Decreto de $\mathbf{1 5}$ 
de diciembre del mismo año. Para la vigilancia en materia de abastos, se creó la Fiscalía de Tasá por Ley de 30 de septiembre de 1940, la que, con su acción vigilante, es la encargada de procurar la normalidad de las actividades en esta materia, castigando las infracciones, con independencia de la Comisaría General de Abastecimientos.

Por Ley de 24 de junio de 1941 , se reorganiza la Comisaría, y se ejecuta la misma por Decreto de $\mathbf{1 1}$ de julio del mismo año.

Bástenos esta pequeña reseña de la legislación fundamental que sobre abastecimientos ha elaborado el Gobierno, para darnos cuenta de la honda preocupación que tal materia ha sido y es para las altas autoridades de la nación, que hoy no sólo afecta a nuestra Patria, sino a todo el mundo. Esta legislación que dejamos reseñada centralizó, desde el primer momento, el abastecimiento de productos, que por su consumo, es de carácter general, con el fin de hacer un reparo de ellos de una manera justa y que evitara tanto el encarecimiento como la ocultación, ya que las economías modestas se sentirian seriamente afectadas por tales causas; y debido a esta necesidad de tipo nacional, le fueron sustraídas a los Ayuntamientos y Alcaldes las facultades y atribuciones que en materia de abastos les estaban conferidas por la Ley y Reglamento municipal vigente, así como por las Ordenanzas municipales; convirtiéndose los Alcaldes en Delegados locales de Abastos, con delegación de los Gobernadores-Delegados provinciales-que a su vez la reciben del Comisario General. Mas a pesar de haberles sustraído a las autoridades locales las facultades que tradicionalmente habían detentado en tal materia, no quedaron completamente carentes de autoridad en tal sentido, sino que sólo les fueron restadas algunas respecto a los artículos intervenidos por la Comisaría General; pero aquellos que no habían sido incluídos en tal intervención, seguían estando sometidos a la autoridad local de una manera autónoma.

Recientemente, por Decreto de 30 de agosto de 1946, se delegaron en los Municipios parte de las facultades concedidas a la Comisaría General de Abastecimientos, en Ley, ya citada, de 24 de junio de 1941, delegación que señala el primer paso en 
la reversión a los Ayuntamientos de sus tradicionales facultades y atribuciones, pues el deseo del Gobierno es el de devolver a las Entidades locales aquellos servicios que han sido y deben ser de su completa competencia, como muy claro lo manifestó el Caudillo: “Es necesario que las Corporaciones provinciales y locales sustituyan al Estado en el detalle de las tareas propias de sus respectivas comarcas y propongan, e incluso en muchos casos resuelvan, sus problemas, para lo que el Estado delegará en ellas las funciones que no puede ni debe centralizar" (31). Pero esto sólo podrá hacerse cuando las circunstancias, que informan las medidas de gobierno, lo permitan.

También en la Ley de Bases de Régimen local de 17 de julio de $\mathbf{1 9 4 5}$ se apuntan las atribuciones que se otorgarán, en futura Ley, en materia de abastos a los Municipios y Alcaldes, pero no estando aún desarrolladas tales Bases en Ley articulada, sólo enumeraremos las que contienen dichas atribuciones: Base 11, "Competencia municipal", apartado $d$ ); Base 12, "De los servicios municipales obligatorios", párrafo $2 .^{\circ}$; Base 18 , "Municipalización de servicios". Estos preceptos dicen claramente la magnitud de la competencia que en la futura Ley se les conferirán a las Corporaciones y Alcaldes respecto al servicio de abastos.

$\mathrm{Y}$ para terminar, permítasenos hacer observar que, a pesar de la modestia del trabajo, queda expueșta la misión y cometido que siempre les ha estado encomendado a las Corporaciones y autoridades locales respecto al abasto público, lo que hace improcedente la creencia que algunas autoridades locales puedan tener, que con las facultades delegadas en los Ayuntamientos por el Decreto de $\mathbf{3 0}$ de agosto último, se ha cargado sobre las Corporaciones una responsabilidad que sólo compete a la Comisaría General de Abastecimientos, cuando la auténtica realidad es bien distinta, como queda expuesto y como indudablemente tendrá que volver a ser cuando las circunstancias lo aconsejen.

\section{Pedro Ponce Llavero}

Licenciado en Derecho.

(31) Discurso del Generalisimo en Valladolid, en 20 de mayo de 1945. 